\title{
Avant-propos
}

\section{Le jeu croisé des dynamiques agraires et foncières}

\author{
L'analyse approfondie de la dynamique du système foncier est indispensable pour comprendre l'évolution du système agraire
} Pierre Milleville ${ }^{1}$

La question du foncier est effectivement une question essentielle pour comprendre les dynamiques qui sont à l'œuvre en ce qui concerne la façon dont les sociétés rurales exploitent leur milieu et gèrent leurs ressources. C'est la raison pour laquelle " Le jeu croisé des dynamiques agraires et foncières " a été retenu comme un des six thèmes abordés lors du Colloque international sur le foncier qui s'est tenu à Montpellier en mai 2006. Ce numéro thématique a pour but de contribuer à la valorisation des travaux de ce colloque en publiant une sélection des communications faites sur ce thème ${ }^{2}$.

L'évolution des règles de gestion du foncier en relation avec la dynamique des systèmes agraires a donné lieu à des interprétations théoriques variées. À l'occasion de ce colloque on s'est efforcé d'analyser de façon empirique, à partir d'études de cas réalisées dans des situations agraires diversifiées, les relations conjointes entre dynamiques foncières et dynamiques agraires afin d'analyser les processus d'interaction entre les faits techniques, les rapports sociaux de production et les pratiques foncières.

En effet, si les règles et les pratiques de gestion des ressources et en particulier du foncier sont dépendantes de l'histoire, des formes d'organisation des sociétés rurales et de leur insertion dans des espaces économiques et sociopolitiques très larges, elles sont également déterminées par les systèmes techniques adoptés pour exploiter le milieu. Mais réciproquement, les formes d'organisation sociale et d'accès aux ressources influent sur ces systèmes techniques. La compréhension des interactions entre les modes d'accès au foncier et les modes d'utilisation agricole des terres permet alors d'éviter l'écueil d'une simple mise à jour des relations entre intensification et appropriation foncière et de révéler au contraire la diversité des droits et des modes de gestion du foncier au sein des territoires.

Les articles retenus pour ce numéro thématique sur le foncier abordent ces interactions entre pratiques agricoles et gestion des terres suivant différents angles d'approche :

- Du système technique aux règles et pratiques de gestion du foncier

Au sein d'un même territoire, la nature variée des milieux biophysiques et des systèmes de culture qui leur correspondent peut influer sur les modes de gestion du foncier. L'analyse des systèmes techniques spécifiques à chacun de ces milieux permet de mieux comprendre la diversité des modes d'accès, d'appropriation et d'utilisation du foncier.

- Des pratiques sociofoncières aux pratiques techniques

Les règles et pratiques sociales qui président à l'allocation des terres peuvent également jouer sur la nature de leur mode de mise en valeur. À l'effet des " terrains " au sens agronomique du terme, peut alors venir s'ajouter l'effet des différenciations sociales dans l'accès aux ressources pour expliquer les différenciations des systèmes techniques.

\section{- Changement technique et dynamiques foncières}

Les processus de changement technique, comme les aménagements hydro-agricoles ou les plantations, concourent à la production de nouvelles règles d'usage et d'accès à la ressource foncière. Ils participent généralement à l'émergence de nouvelles formes de différenciation sociale.

L'accroissement de la pression foncière que l'on observe dans de nombreux pays du Sud constitue un facteur déterminant des dynamiques agraires qui y sont à l'œuvre. Pour être positive cette dynamique nécessite une adaptation des règles qui président à la gestion du foncier. Les différents articles publiés dans ce numéro montrent clairement la grande diversité de situations à laquelle on est confronté dès lors que l'on cherche à faire évoluer ces règles. Cette diversité plaide en faveur d'approches plus circonstanciées et moins dogmatiques dans l'élaboration de nouveaux modes de gestion du foncier, enjeu essentiel pour le développement que ce soit vis-à-vis de la lutte contre la pauvreté ou l'amélioration des performances des agricultures comme le souligne Jean-Pierre Chauveau dans son éditorial.

Philippe Jouve Institut des régions chaudes <jouve@cnearc.fr>

\footnotetext{
${ }_{1}^{1}$ Milleville P. Une agronomie à l'œuvre. Paris : éditions Arguments, éditions Quae, 2007.

${ }^{2}$ Voir ci-après la liste des ouvrages et publications qui valorisent les communications faites sur les autres thèmes du colloque.
} 